\title{
Variable and reversible quantum structures on a single carbon nanotube
}

\author{
Ç. Kılıç, ${ }^{1}$ S. Ciraci, ${ }^{1}$ O. Gülseren, ${ }^{2,3}$ and T. Yildirim ${ }^{2}$ \\ ${ }^{1}$ Physics Department, Bilkent University, Ankara, Turkey \\ ${ }^{2}$ NIST Center for Neutron Research, National Institute of Standards and Technology, Gaithersburg, Maryland 20899 \\ ${ }^{3}$ Department of Materials Science, University of Pennsylvania, Philadelphia, Pennsylvania 19104
}

(Received 9 October 2000)

\begin{abstract}
The band gap of a semiconducting single wall carbon nanotube decreases and eventually vanishes leading to metalization as a result of increasing radial deformation. This sets in a band offset between the undeformed and deformed regions of a single nanotube. Based on the superlattice calculations, we show that these features can be exploited to realize various quantum well structures on a single nanotube with variable and reversible electronic properties. These quantum structures and nanodevices incorporate mechanics and electronics.
\end{abstract}

Unusual properties of electrons in the quantum structures which were realized by using semiconductor heterostructures $\left(A_{n} B_{m}\right)$ have initiated several fundamental studies. ${ }^{1}$ Owing to the band offsets of the semiconductor heterostructures, the energies of the band states of one semiconductor $B$ may fall into the band gap of the adjacent semiconductor $A$. According to the effective mass approximation (EMA), the height (depth) of the conduction (valence) band edge of $A$ from that of $B, \Delta E_{C}\left(\Delta E_{V}\right)$, behaves as a potential barrier for electrons (holes). For example, $m$ layers of $B$ between $n$ layers of two $A$ 's form a quantum well yielding confined electronic states. The depth of the well and the width of the barrier and well (in terms of number of layers $n$ and $m$, respectively) are crucial parameters to monitor the resulting electronic properties. Multiple quantum well structures (MQW's) or resonant tunneling double barrier structures (RTDB's) can be tailored from the combination of $A$ and $B$, and from various stacking sequence of $n$ and $m$.

Single wall carbon nanotubes ${ }^{2}$ (SWNT's) can display metallic or semiconducting character depending on their chiralities and diameters. ${ }^{3,4}$ Similar to the aforementioned idea exploited extensively in crystals, ${ }^{1}$ quantum structures can also be produced in SWNT's. ${ }^{5-9}$ It has been experimentally demonstrated that a rectifying behavior can be achieved by the junction of two different SWNT's. ${ }^{6}$ Furthermore, the transport measurements on the ropes ${ }^{7}$ and individual nanotubes ${ }^{8}$ have indicated a resonant tunneling behavior. Recently, the quantum dot behavior has been also observed. ${ }^{9}$ Like the semiconductor heterostructures, a different electronic property requires each time the fabrication of a new device using SWNT junctions.

In this work, we propose a practical and interesting alternative, and show that various quantum structures can easily be realized on an individual SWNT, and their electronic properties can be variably and reversibly monitored. We predict and use the feature that the band gap of a semiconducting SWNT can be modified by radial deformation ${ }^{10}$ as described in Fig. 1(a). More importantly, if such a deformation is not uniform but has different strength at different zones, each zone displays different band gap. Owing to the band offsets at the junction of different zones, MQW's or RTDB's of the desired electronic character can be formed, and novel electronic nanodevices can be engineered on a single nanotube. This scheme is quite different from the pre- vious constructions of SWNT heterostructures or quantum dots, ${ }^{5}$ where one had to fabricate each time a different junction or topological defects to satisfy the desired electronic character.

First-principles calculations are carried out within the generalized gradient approximation (GGA) using plane waves (PW) with a cutoff energy of $500 \mathrm{eV}$ and ultrasoft pseudopotentials. ${ }^{11,12}$ Constrained structure optimizations are performed on the SWNT under transversal compression. The zigzag $(7,0)$ tube is a semiconductor when it is undeformed,

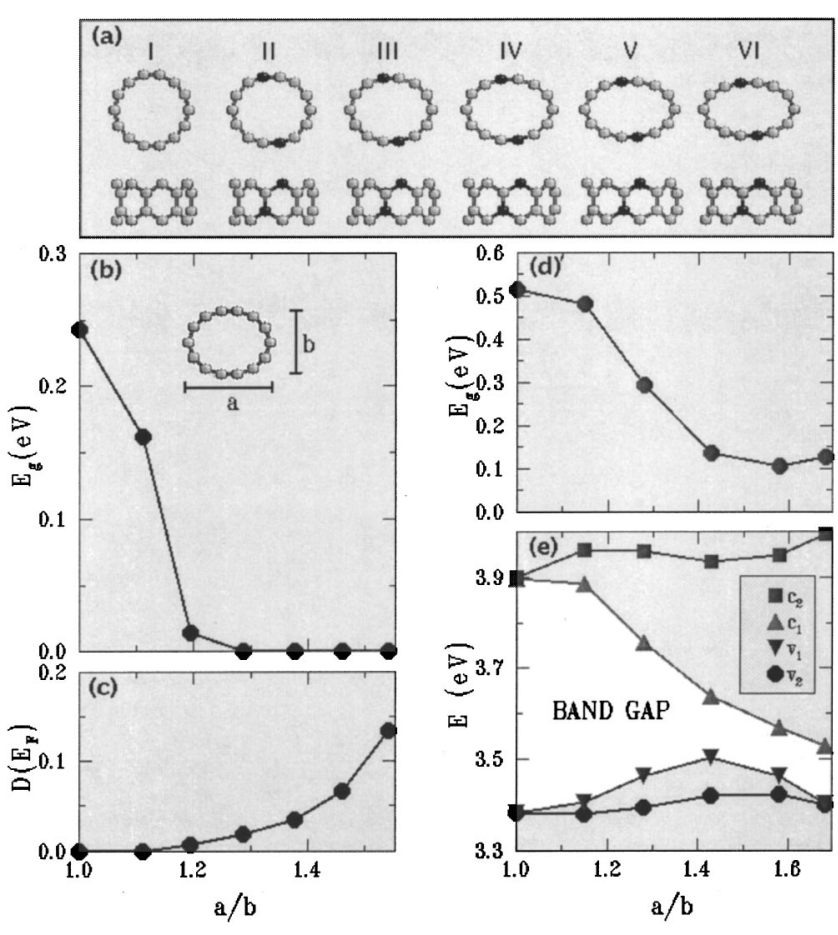

FIG. 1. (a) Top and side view of (primitive) unit cells of the $(7,0)$ SWNT under different degrees of elliptic (circumferential) deformation. Undeformed tube with circular cross section is labeled type I. (b) Variation of the energy band gap $E_{g}$, and (c) density of states at the Fermi energy, $D\left(E_{F}\right)$ with deformation $a / b$ calculated by the first-principles PW method. (d) Variation of $E_{g}$, and (e) first and second states at the edge of the conduction band $\left(c_{1}, c_{2}\right)$, and first and second states at the edge of the valence band $\left(v_{1}, v_{2}\right)$ with $a / b$ calculated by the TB method. 
but its band gap $E_{g}$ is modified upon introducing an elliptic deformation, which is quantified by the ratio of the elliptic major axis to the minor axis $a / b$. The band gap decreases with increasing $a / b$, and eventually vanishes for $a / b \sim 1.28$ [see Fig. 1(b)]. After the onset of metalization upon the closure of the band gap, the density of states at the Fermi energy $D\left(E_{F}\right)$ increases with further increase of $a / b$, as shown in Fig. 1(c). Relatively stronger radial deformations are required for the closure of the gap of $(8,0)$ and $(9,0)$ tubes. The radial deformation $a / b$, the strain energy per atom $E_{s}$, and the compressive force on the fixed atom $F_{c}$ at the closure of the band gap are calculated to be $\left(a / b=1.28 ; \quad E_{s}=18 \mathrm{meV} ; \quad F_{c}=0.32 \mathrm{eV} / \mathrm{A}\right), \quad(a / b=1.54 ;$ $\left.E_{s}=38 \mathrm{meV} ; \quad F_{c}=0.31 \mathrm{eV} / \mathrm{A}\right)$, and $\left(a / b=1.36 ; \quad E_{s}=18\right.$ $\left.\mathrm{meV} ; F_{c}=0.25 \mathrm{eV} / \mathrm{A}\right)$ for $(7,0),(8,0)$, and $(9,0)$ tubes, respectively. ${ }^{12}$ The armchair $(6,6)$ SWNT maintains the metallic behavior despite the radial deformation. Remarkably, the induced deformations, in particular those causing to insulator-metal transition, are elastic. Our results confirm that the atomic structure and hence the electronic properties return to the original, undeformed state when the compressive stress is lifted.

Normally, as the radius $R \rightarrow \infty$ the electronic structure of a SWNT near the band edges becomes similar to that obtained by folding the $\pi^{*}$ and $\pi$ bands of graphene. However, a singlet state at the band edge of a $(n, 0)$ SWNT with small $R$ involves significant $\sigma^{*}-\pi^{*}$ hybridization. ${ }^{3}$ This state occurs above the $\pi^{*}$ band $(n=9)$, but falls in the gap $(n=7,8)$ and eventually closes the gap $(n=6)$ as $n$ decreases., ${ }^{3,12}$ Apparently, the estimate of $E_{g}$ based on the simple extrapolation using the experimental gaps of SWNT with relatively larger radius ${ }^{4}$ is not valid for the $(7,0)$ tube. In the present case, introducing radial deformation and hence increasing the curvature at both ends of the elliptic major axis reduces the band gap of the $(7,0)$ SWNT owing to the enhanced $\sigma^{*}-\pi^{*}$ hybridization.

We also perform tight binding (TB) total energy and electronic structure calculations for the undeformed and uniformly deformed $(7,0)$ SWNT by using transferable parameters $^{13}$ related to carbon $2 s$ and $2 p_{x, y, z}$ orbitals. The radial deformation has been induced by approaching two atoms of the unit cell at one end of the diameter to two similar atoms at the other end indicated by dark atoms in Fig. 1(a). Once the transversal strain is set by fixing these four atoms, the rest of the atoms are relaxed by the conjugate gradient method. We consider undeformed $(a / b=1)$ and five different degrees of radial deformation $(a / b>1)$. The variation of $E_{g}$, first and second states of the conduction band, $\left(c_{1}\right.$ and $\left.c_{2}\right)$ and those of the valence band, $\left(v_{1}\right.$ and $\left.v_{2}\right)$ are illustrated in Figs. 1(d) and 1(e). Data points on the curves shown in these figures correspond to different degrees of elliptic deformations from I to VI as described in Fig. 1(a). We note deviations between the first-principles PW and empirical TB results perhaps due to the differences in the details of the deformations and limitations of the empirical method. It is also known that the band gap $E_{g}$ is usually underestimated by local density approximation (LDA) calculations. On the other hand, as discussed before, the $\sigma^{*}-\pi^{*}$ hybridization effect is crucial for setting $E_{g}$, and first-principles PW calculations described it better. While the present GGA calculations for the undeformed $(7,0)$ tube finds $E_{g}=0.242 \mathrm{eV}$, an earlier LDA calculation ${ }^{3}$

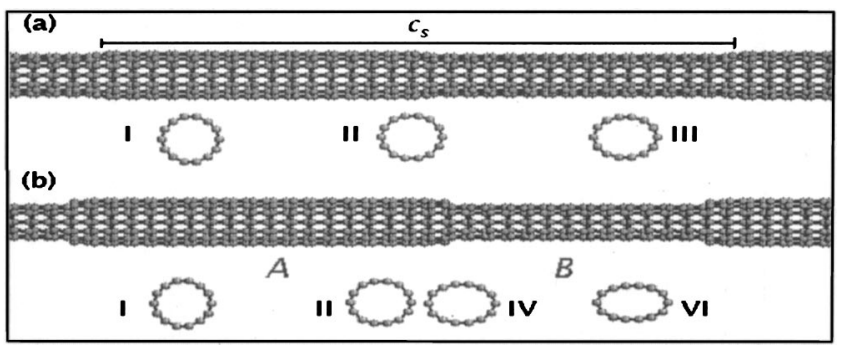

FIG. 2. Schematic descriptions of the $\left(A_{8} B_{8}\right)$ supercells of the superlattices generated from the $(7,0)$ SWNT. $c_{s}$ is the lattice parameter. (a) Region $A$ is undeformed, $B$ has elliptic cross section of type III. The interface layer has type-II deformation. (b) Severely deformed $B$ has type-VI deformation with two interface layers of type-II and -IV deformation.

predicted $E_{g}=0.09 \mathrm{eV}$. Our TB calculations using transferable parameters predict $E_{g} \sim 0.5 \mathrm{eV}$ and hence relatively stronger deformation is required to reduce the TB gap $E_{g}$ to $0.1 \mathrm{eV}$. Earlier TB calculations found $E_{g} \sim 1.0 \mathrm{eV}$ for undeformed tube. ${ }^{14}$ Comparison of band gaps measured by STM spectroscopy ${ }^{6}$ with those calculated by different methods, and an extensive analysis for their variation with the radial deformation applied on different SWNT's will be presented elsewhere. ${ }^{12}$ Nevertheless, both methods (PW and TB) predict here similar overall behavior for the band gap variation with the radial deformation. We will use the TB method to study MQW's, since it allows us to treat a large number of carbon atoms, which cannot be treated easily with the present first-principles PW method. We will treat the $(7,0)$ tube as a prototype system.

Reducing $E_{g}$ and eventually the onset of insulator-metal transition, and further metallization of certain SWNT's with increasing radial deformation, and the reversible nature of all these sequence of physical events incorporate important ingredients suitable to form quantum structures and nanodevices. We investigate MQW's as a generic system and demonstrate that one can generate electronic properties convenient for various device applications. To start, we consider a $(7,0)$ zigzag nanotube, that is pressed to squash only at certain regions. We assume that the undeformed region $A$ of $n$ unit cells, ${ }^{15}$ and adjacent deformed region $B$ of $m$ unit cells (one interface atomic layer at both side has intermediary deformation) repeat periodically, so that the translational periodicity along the axis of the tube involves $n+m=16$ cells and 448 carbon atoms. This tube forms a $\left(A_{n} B_{m}\right)$ superlattice of semiconductor heterostructure, where the band gap of $A$ is larger than that of $B$. Since $E_{g}(A ; a / b=1)$ $>E_{g}(B ; a / b>1)$, a band offset shall occur at the junction. The $(n=8, m=8)$ supercells of the superlattices are schematically described for two different degrees of deformation in Fig. 2. Note that at low degree of deformation the junction can be formed by using one interface layer, while more interface layers may be necessary if $B$ is severely deformed or a graded junction is aimed.

Experimental and theoretical methods have been proposed in the past to determine the band offsets, and hence to reveal the band diagram perpetuating along the superlattice axis. For the present situation ambiguities exist in calculating alignments of the band edges and to determine band diagram 

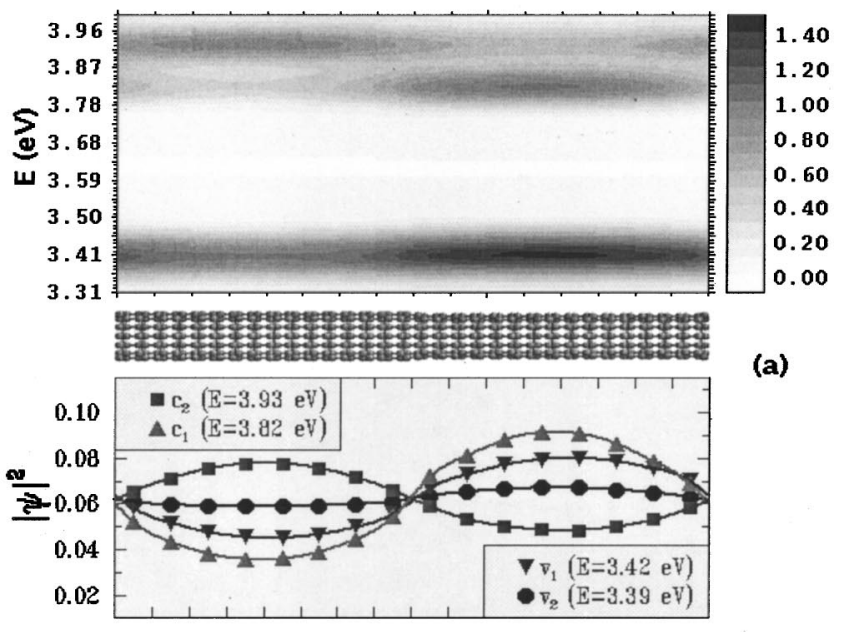

(a)
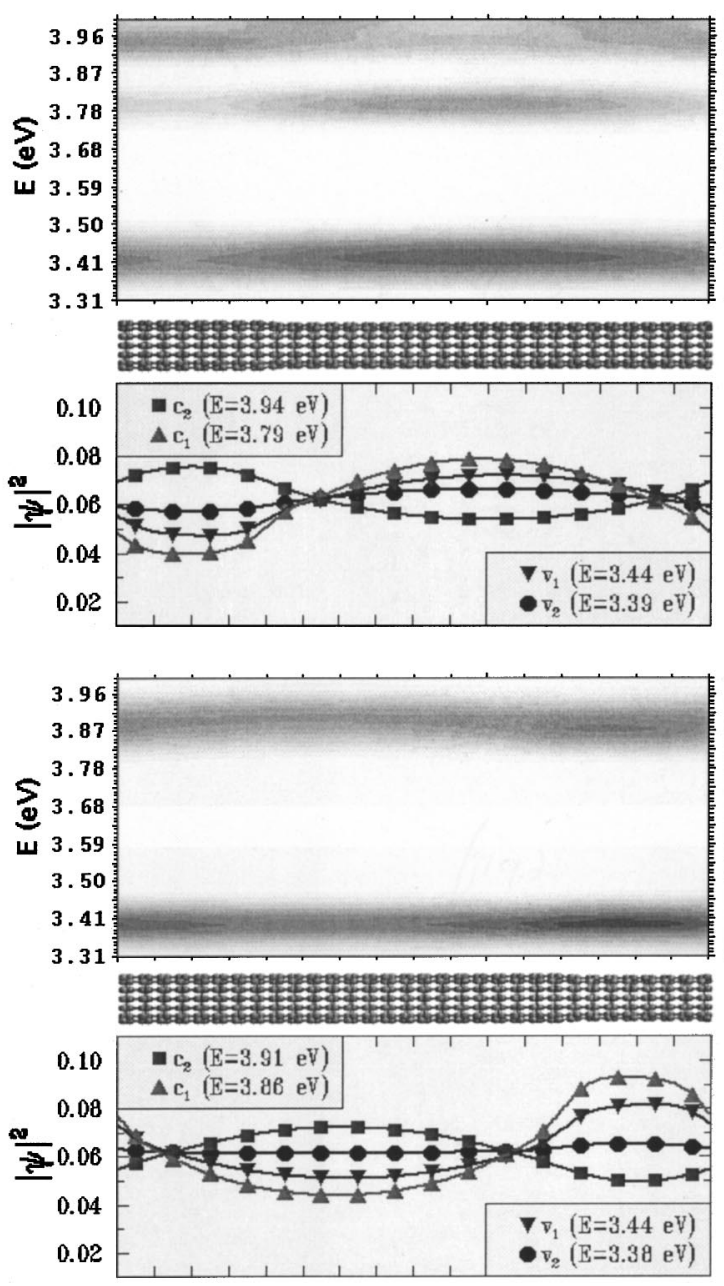

FIG. 3. Upper panel: local density of states $\mathcal{L}(E, j)$; lower panel: the state density $\left|\Psi_{i, \mathbf{k}}(j)\right|^{2}$ at the cell $j$ of the superlattices which display MQW's behavior. (a) $\left(A_{8} B_{8}\right)$; (b) $\left(A_{4} B_{12}\right)$; (c) $\left(A_{12} B_{4}\right)$.

in real space including band bowing due to the charge transfer between $A$ and $B$. Even if the band diagram were known, it is not obvious whether EMA is applicable for an individual, non uniformly deformed SWNT. Therefore, instead of applying EMA to the 1D band diagram, we directly calculate the electronic structure of the $\left(A_{n} B_{m}\right)$ superlattice on an individual $(7,0)$ SWNT. The band alignment is not ex-

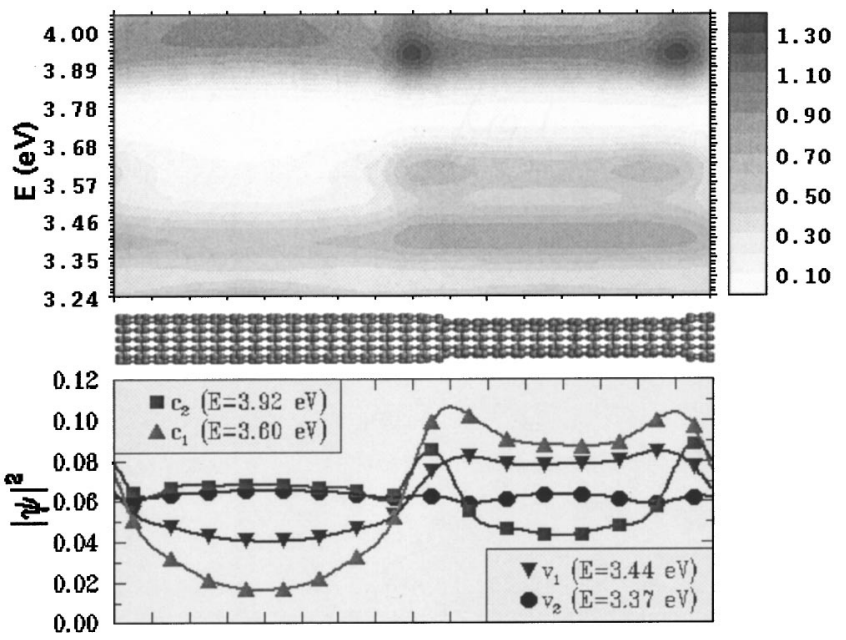

FIG. 4. The same as Fig. 3(a) except that $B$ has type-VI cross section shown in Fig. 1(a).

plicit, but it is indigenous to the method and hence the confined states shall be obtained directly from the present TB superlattice calculations. $^{16}$

We performed calculations on three different superlattices described in Fig. 2(a), i.e., $\left(A_{8} B_{8}\right) ;\left(A_{4} B_{12}\right) ;\left(A_{12} B_{4}\right)$, and calculated the electronic states $\Psi_{i, \mathbf{k}}(\mathbf{r})$ with band energy $E_{i, \mathbf{k}}$. Here, $i$ and $\mathbf{k}$ are the band index and wave vector of the superlattice along its axis. Because of flat superlattice bands, we considered only the $\Gamma$ point in the superlattice Brillouin zone. Figure 3 illustrates the local (or cell) density of states, $\mathcal{L}(E, j)=\sum_{i, \mathbf{k}} \int_{j} d \mathbf{r}\left|\Psi_{i, \mathbf{k}}(\mathbf{r})\right|^{2} \delta\left(E-E_{i, \mathbf{k}}\right)$ and the state density $\left|\Psi_{i, \mathbf{k}}(j)\right|^{2}=\int_{j} d \mathbf{r}\left|\Psi_{i, \mathbf{k}}(\mathbf{r})\right|^{2}$ both integrated at each cell $j$ in the supercell. $\mathcal{L}(E, j)$ with higher density near the band edges of $B$ (i.e., small gap region) is due to quantum well states and hence is consistent with the discussion presented at the beginning. Second peak of $\mathcal{L}(E, j)$ in the conduction band occurs in $A$, and becomes well separated from the first peak in the superlattice $\left(A_{4} B_{12}\right)$. The well known behavior of MQW's is apparent with the confinement of states at the band edges. The first states at the band edges of $B$, i.e., $c_{1}$ and $v_{1}$ are confined in $B$ suggesting a normal band offset. The confinement of the second state in the valence band, $v_{2}$ is rather weak. On the other hand, the second state of the conduction band, $c_{2}$ is not confined in the well of $B$, but is localized at the barrier of $A$. It appears that the energy of $c_{2}$ occurs above the well in $B$, and $c_{2}$ cannot match with the next higher energy state of $B$. Similar to that observed in short periodicity $\mathrm{Al}_{x} \mathrm{Ga}_{1-x} \mathrm{As}$ superlattices, ${ }^{16}$ this situation demonstrates that the description of the superlattice electronic structure in terms of one-dimensional (1D) multiple square well states obtained within the simple EMA can fail owing to the band structure effects. The confinement of $c_{1}$ and $v_{1}$ states increases with $n$, i.e., with the length of the barrier region. This is an expected result, since the longer barrier prevents the tunneling of these states through $A$. Also the energy of $c_{1}$ raises with decreasing $m$. This is a direct consequence of the uncertainty principle.

Figure 4 shows the MQW's behavior of the superlattice described in Fig. 2(b), where $B$ is strongly deformed. The state density and local density of states indicate that the confined states $c_{1}$ and $v_{1}$ display relatively higher localization at 
the interface. This situation originates from the interface atomic structure connecting $A$ to strongly deformed $B$. Different band offsets can also be realized by setting up different level of deformations at both $A$ and $B$. Again, depending on the level of the deformation $E_{g}(B ; a / b>1)$ can even be zero that makes a metal-semiconductor superlattice structure. Furthermore, from the junction of two metalized SWNT's having different $D\left(E_{F}\right)$ one can form a metal-metal superlattice.

In MQW's, the truly 1D states are normally propagating with the wave vector $\mathbf{k}$, and form a band structure. The bands become flatter with increasing $n$, and eventually the band picture breaks down and states become totally localized in the quantum well (or in $B$ ). This way the superlattice is expected to experience a Mott metal-insulator transition. Furthermore, a randomly deformed SWNT can be an interesting system to investigate electron localization in $1 \mathrm{D}$. The modulating or $\delta$ doping of a MQW's or QW's (also quantum dots) may exhibit interesting effects on the transport properties. ${ }^{16}$ It is interesting to note that the resonance condition of a RTDB's with $A_{n} B_{m} A_{n^{\prime}}$ having contacts to metal reservoirs from both ends shall be monitored by the defor- mation and size of $B$. Strain or pressure nanogauges or variable nanoresistors can be developed based on the fact that the metalization and hence the conductance of a $(7,0)$ nanotube can be changed with the applied deformation. Also a junction $A_{n^{\prime}} B_{m}$, with metallic $B$ is expected to show a rectifying behavior. We also note that a 3D grid of MQW's can be constructed by periodic stacking of tubes where quantum wells occur at crossing points. The electronic properties of this system can be varied with the stacking sequence and applied pressure. Finally, we point out that the recent experimental work ${ }^{17}$ which showed that the controlled local deformation can be achieved.

In conclusion, we showed that the electronic properties of a semiconducting SWNT can be modified by introducing radial deformation which can be used to produce interesting quantum structures and devices on a single tube, such as MQW's, RTDB's, rectifying junction, and variable nanoresistor with continuously tunable electronic properties.

This work was partially supported by the National Science Foundation under Grant No. INT97-31014 and TÜBITAK under Grant No. TBAG-1668(197 T 116).
${ }^{1}$ Highlights in Condensed Matter Physics and Future Prospects, edited by L. Esaki, Volume 285 of NATO Advanced Science Institute (Plenum, New York, 1991).

${ }^{2}$ S. Iijima, Nature (London) 354, 56 (1991).

${ }^{3}$ X. Blase et al., Phys. Rev. Lett. 72, 1878 (1994).

${ }^{4}$ J. W. G. Wildöer et al., Nature (London) 391, 59 (1998); T. W. Odom et al., ibid. 391, 62 (1998).

${ }^{5}$ L. Chico et al., Phys. Rev. Lett. 76, 971 (1996); 81, 1287 (1998); R. Saito et al., Phys. Rev. B 53, 2044 (1996); J.-C. Charlier et al., ibid. 53, 11108 (1996).

${ }^{6}$ P. G. Collins et al., Nature (London) 278, 100 (1997).

${ }^{7}$ M. Bockrath et al., Science 275, 1922 (1997).

${ }^{8}$ S. J. Tans et al., Nature (London) 386, 474 (1997).

${ }^{9}$ A. Bezryadin et al., Phys. Rev. Lett. 80, 4036 (1998).

${ }^{10}$ The $\sigma^{*}-\pi^{*}$ hybridization effects in Ref. 3 and the experimental results in Ref. 9 have led to the band gap modifications by radial deformation in the following studies: A. Rochefort et al., Chem. Phys. Lett. 297, 45 (1998); Phys. Rev. B 60, 13824 (1999); C. Kilic, Ph. D. thesis, Bilkent University, 1999 (unpublished); C-J.
Park et al., Phys. Rev. B 60, 10656 (1999); M. S. C. Mazzoni et al., Appl. Phys. Lett. 76, 1561 (2000); L. Liu et al., Phys. Rev. Lett. 84, 4950 (2000); P. E. Lammert et al., ibid. 84, 2453 (2000); L. Yang and J. Han, ibid. 85, 154 (2000).

${ }^{11}$ First-principles calculations were performed by planewave code CASteP; M. C. Payne et al., Rev. Mod. Phys. 64, 1045 (1992). In the present calculations we used Monkhorst-Pack special points with $2 \times 2 \times 20 \mathbf{k}$ points mesh, and the total energies converged within $0.5 \mathrm{meV} / \mathrm{atom}$.

${ }^{12}$ O. Gülseren et al., (unpublished).

${ }^{13}$ C. H. Xu et al., J. Phys.: Condens. Matter 4, 6047 (1992).

${ }^{14}$ N. Hamada et al., Phys. Rev. Lett. 68, 1579 (1992); V. H. Crespi and M. L. Cohen, ibid. 79, 2093 (1997).

${ }^{15}$ A cell denotes the primitive unit cell of the $(7,0)$ nanotube, that has 28 carbon atoms. The $A_{8} B_{8}$ supercell contains 16 such cells.

${ }^{16}$ S. Ciraci et al., Phys. Rev. Lett. 58, 2114 (1987); Phys. Rev. B 36, 1225 (1987); 38, R12 728 (1988).

${ }^{17}$ T. W. Tombler et al., Nature (London) 405, 769 (2000). 